\title{
Effect of Presence of Human Body on Antenna Gain
}

\author{
Asma Lak ${ }^{1}$, Naser Parhizgar² and Mahbobeh Lak ${ }^{1}$ \\ 'Department of Electrical Engineering, College of Engineering, Genaveh Branch, Islamic Azad University, Genaveh, \\ Iran; Lak.asmae@gmail.com \\ 2Department of Electrical Engineering, College of Engineering, Shiraz Branch, Islamic Azad University, Shiraz, Iran
}

\begin{abstract}
This paper focuses on the interaction between two types of antenna (Slot Coupled Patch Antenna and Probe Feed Patch Antenna) and human head model at 835MHz. Real experiments on human body and electromagnetic fields is not possible because of it the HFSS software has been used to simulate the head model and antenna as an exposure source. The simulation results (calculated in SAR and E field strength parameters) show that the electromagnetic fields can be absorbed in human tissues. Regular papers have been done in this way. In this paper it is shown that the human tissues can decrease the gain of antenna as an interaction.
\end{abstract}

Keywords: Antenna Gain, Biological Effects, HFSS Software, Human Head Model, Probe Feed Patch Antenna, Slot Coupled Patch Antenna

\section{Introduction}

Many researchers all of the world work on EMFs (Electromagnetic Fields) and their applications ${ }^{1,2}$. The effects are various and divide into thermal and nonthermal and also these effects depend on many parameters such as exposure source, geometry of human tissues, frequency of fields and etc. Human tissues have electrical properties ${ }^{3}$ and because of it the electromagnetic fields can interact with them in three forms: absorption, reflection or transmission. The various mechanisms have been introduced for these interactions by many researchers. If electromagnetic passes from human tissue, it can be converted to heat and damage the body because the temperature has been rise ${ }^{4,5}$.

\section{High Frequency Electromagnetic Fields}

Human body has various tissues with various dielectric properties. Also the electrical properties on different frequencies have different values, so the biological effects on various frequencies are different ${ }^{6}$. The mechanisms are heating and induce electric and magnetic currents. Eye damage is including cornea injury, cataract and retina, lens and iris effects ${ }^{7-10}$.

Skin damages are including (increase the temperature of the skin and burning, deep burns, heat exhaustion and heat stroke) ${ }^{11}$.

Blood-Brain Barrier (BBB) $)^{12-14}$, RF Haring (effect on hearing $)^{15}$, Behavioral effects including loss, sleep disorders and insomnia, decrease in REM sleep, slowed motor skills and raction time in school children, impaired nervous system activity, loss of concentration and "fuzzy thinking", spatial disorientation, change in the brain's electrical activity ${ }^{14-16}$, decreased immune function, increased heart rate, increased blood pressure, DNA damage (genetic damage) and changes in DNA repair capacity, cell proliferation and cancer ${ }^{14,17-19}$.

\section{Discussion}

The human head model and antenna in the commercial systems are modeled. Usually the phantoms are human

*Author for correspondence 
body shaped shells with low $\varepsilon$ and losses. The materials with the same human body characteristic (the same $\varepsilon, \sigma$, $\mu$ ) have been placed in the phantoms and situated near the antenna as an exposure source. Then the SAR and E-field strength values are calculated to show that if the exposure device is standard or not. (SAR is a unit for calculate the value of EM absorption in tissues. This parameter express in terms of $(\mathrm{Watt} / \mathrm{Kg}))$. The most important parameters in SAR calculations are field strength, frequency, exposure environment and etc ${ }^{2}$.

The experiment with human body is not possible because it is dangerous for human body so in the engineering fields the antenna and human body should simulate. In this article the HFSS software is used.

The human head as the most important part of body next to cell phone are modeled. Also the probe feed patch antenna, slot coupled patch antenna and dipole antenna as an exposure sources are simulated. All of the simulations are in $835 \mathrm{MHz}$ because it is a useable frequency in mobile telecommunication systems.

The human head model i.e. phantom that is including three layers (Skin, Skull, Brain) and a shell around them. All of these tissues have different electrical properties including permittivity and conductivity. Both of these properties are varying with frequency. The electrical characteristics of human head are in Table 1.

Table 1. Dielectric property and conductivity of body tissues at the frequencies $835 \mathrm{MHz}$

\begin{tabular}{|l|c|c|c|}
\hline Tissue & $\varepsilon_{\mathrm{r}}$ & $\boldsymbol{\sigma}$ & $\begin{array}{c}\text { Thickness of } \\
\text { tissues }(\mathrm{mm})\end{array}$ \\
\hline Skull & 17.40 & 0.25 & 105.1 \\
\hline Skin & 35.40 & 0.63 & 106.5 \\
\hline brain & 45.26 & 0.92 & 98.3 \\
\hline Shell & 4.6 & 0 & 111.5 \\
\hline
\end{tabular}

\section{Simulations}

In this part a three layer human head model according to Table 1 has been simulated that shown in Figure 1.

To complete the model, two antennas have been simulated; Probe feed patch antenna and slot coupled patch antenna that has been shown in Figure 2 and 3. All of the antenna structure characteristics are in reference 20 and $21^{20,21}$.

All of the simulations have been done at $835 \mathrm{MHz}$ and by HFSS software.
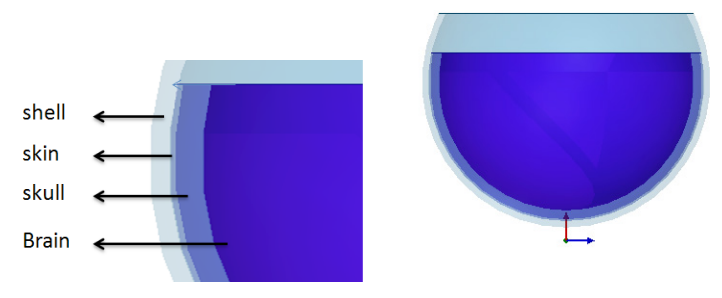

Figure 1. Three layer human head model in HFSS.

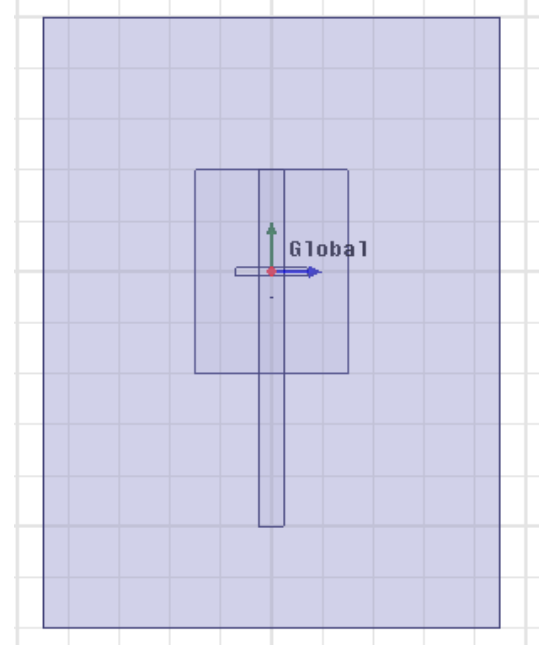

Figure 2. Slot coupled patch antenna.

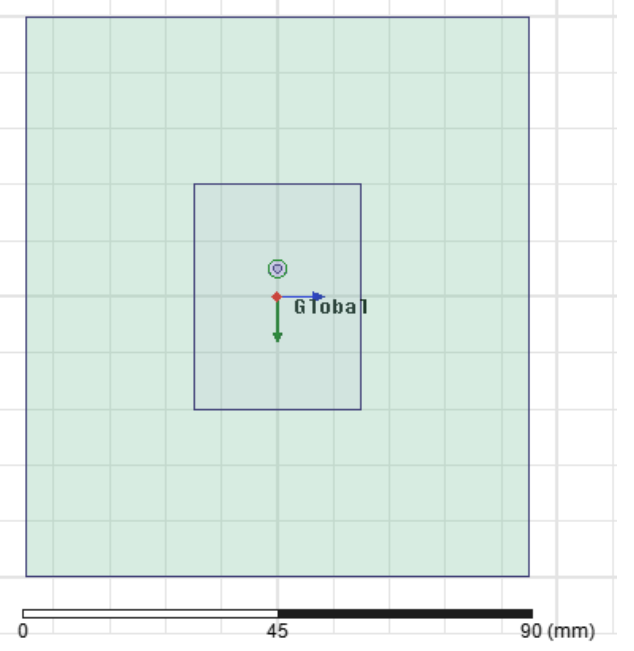

Figure 3. Probe feed patch antenna.

\section{Results}

Because of dielectric properties of human tissues, it can be interact with electromagnetic fields. Duo to it, in the first step the E-field strength has been calculated to show the level of absorption of EM field in human head model. In the commercial model the human head are situated 
next to the antenna to test. In this section this model has been simulated with two antennas. The results for SAR and E-strength have been shown in Figures 4-13.

\subsection{Slot Coupled Patch Antenna (Figures 4-8)}
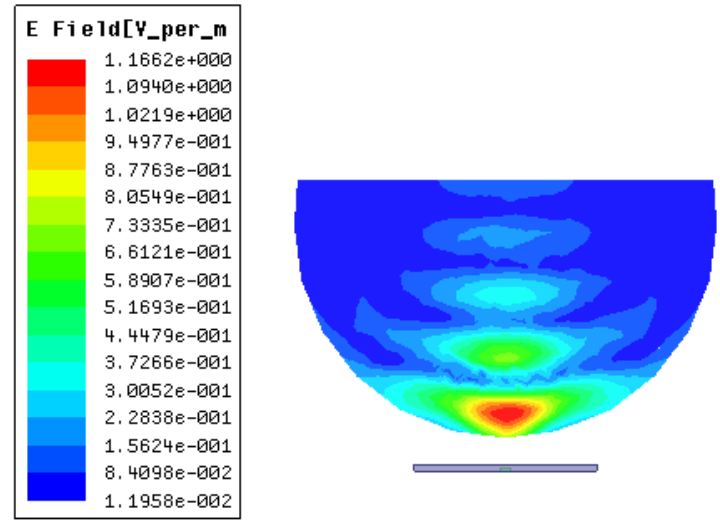

Figure 4. The E-field strength in brain layer.

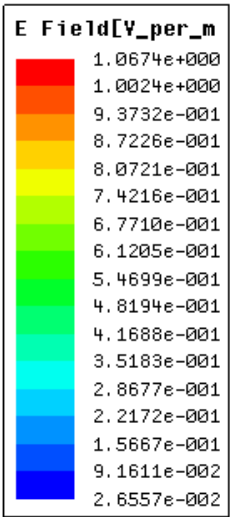

Figure 5. The E-field strength in skull layer.
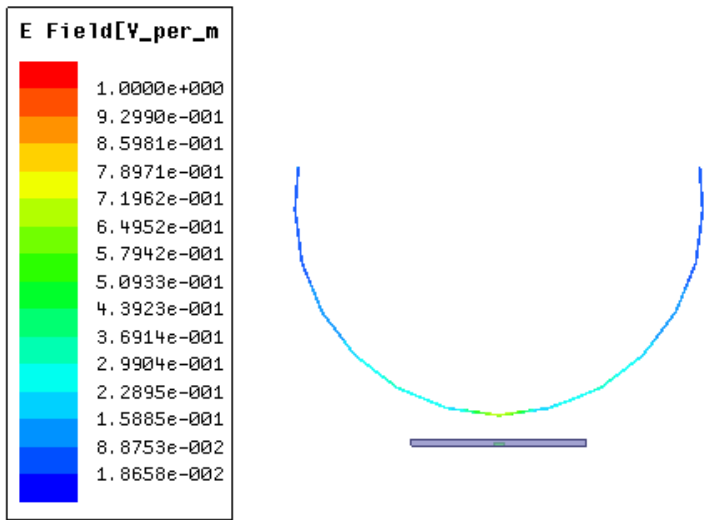

Figure 6. The E-field strength in skin layer.

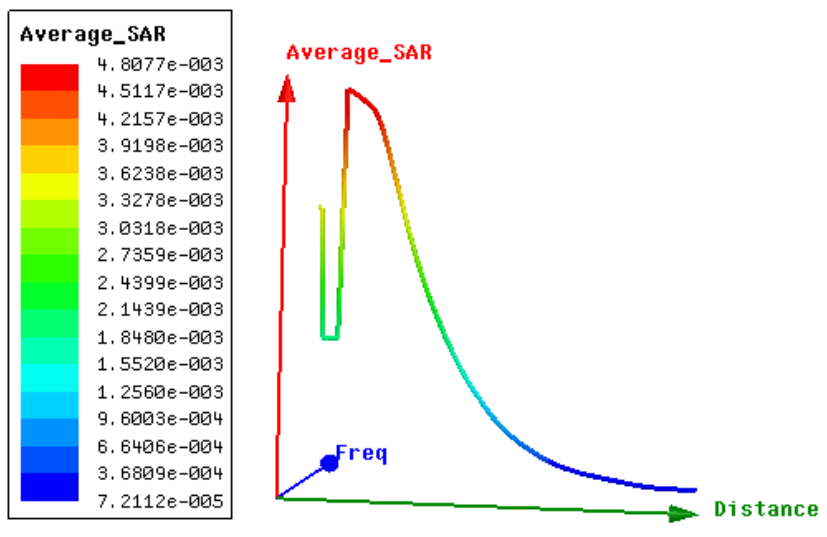

Figure 7. The average SAR.
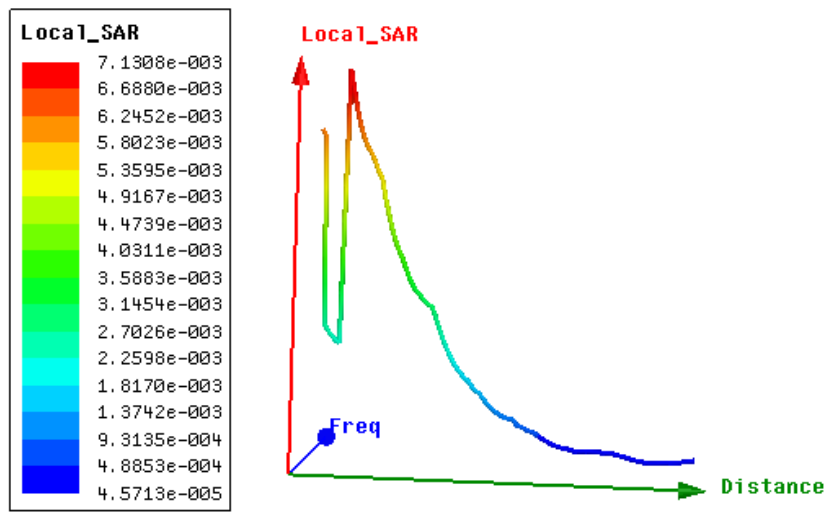

Figure 8. The local SAR.

\subsection{Probe Feed Patch Antenna (Figures 9-13)}
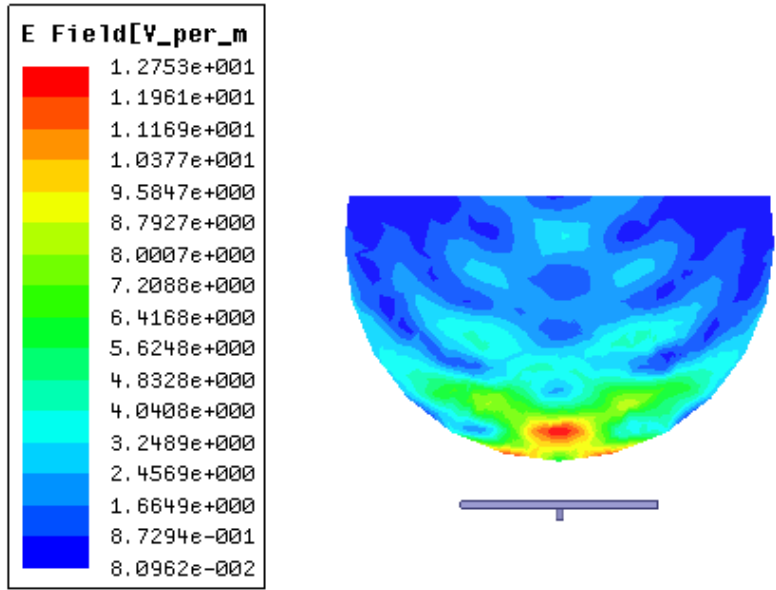

Figure 9. The E-field strength in brain layer. 

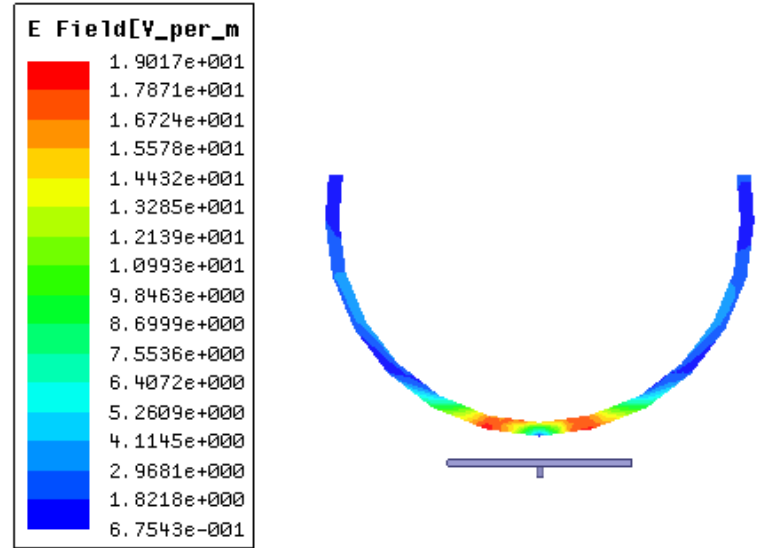

Figure 10. The E-field strength in skull layer.
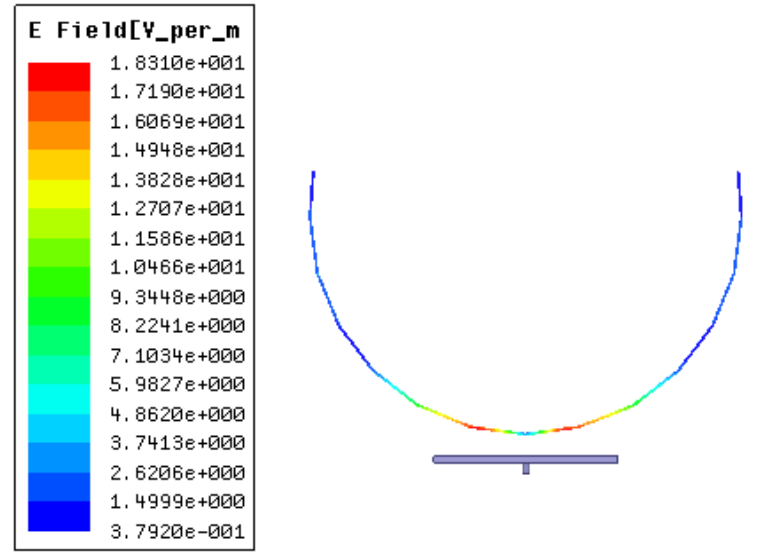

Figure 11. The E-field strength in skin layer

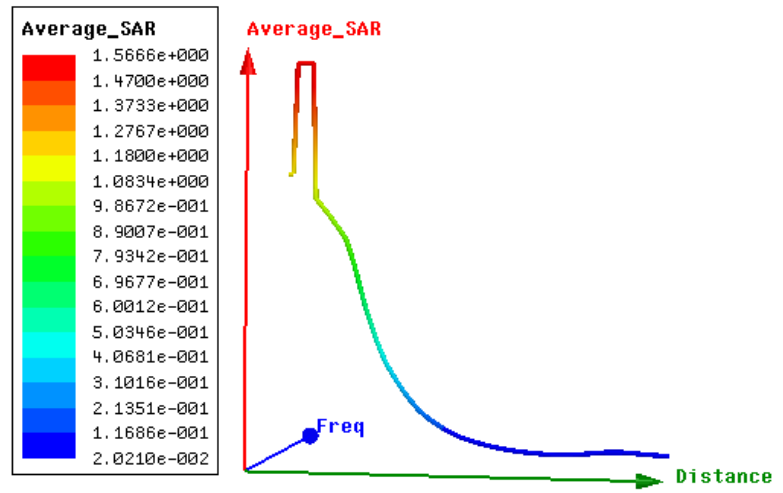

Figure 12. The average SAR.

\subsection{Antenna Gain}

When the word interaction is used, the action is done in both ways. While an antenna affect on tissues, the tissues affect antenna parameters. For example in this paper antenna gain has been evaluated for two types of antenna and the results have been shown in Figures 14-17.

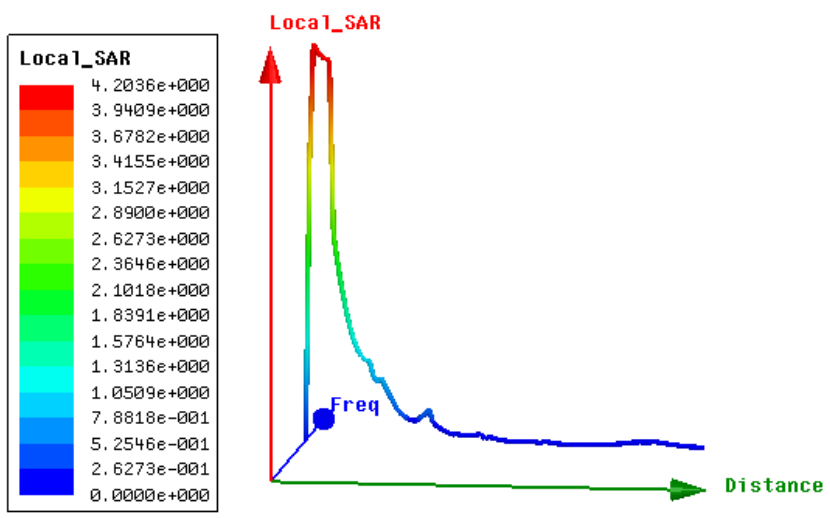

Figure 13. The local SAR.

\subsubsection{Slot Coupled Patch Antenna (Figures 14,15)}
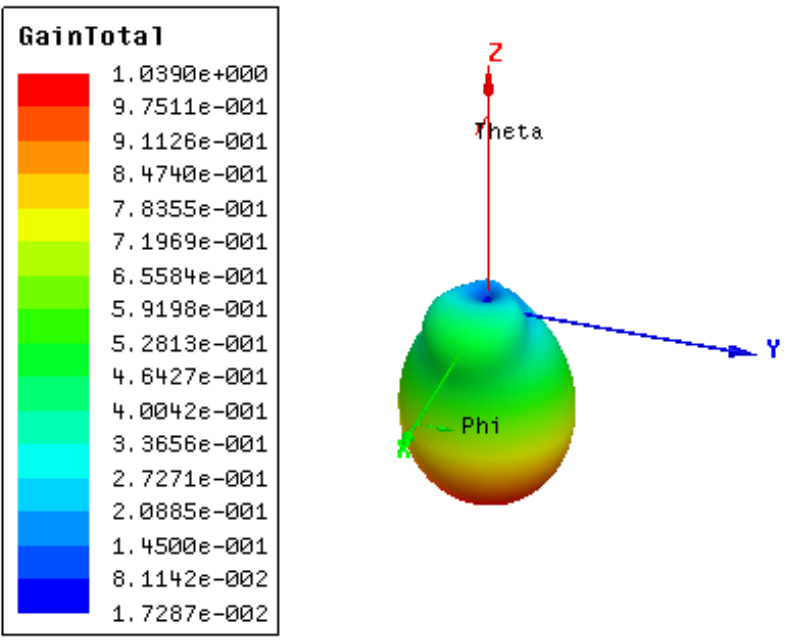

Figure 14. Antenna gain in presence of head.
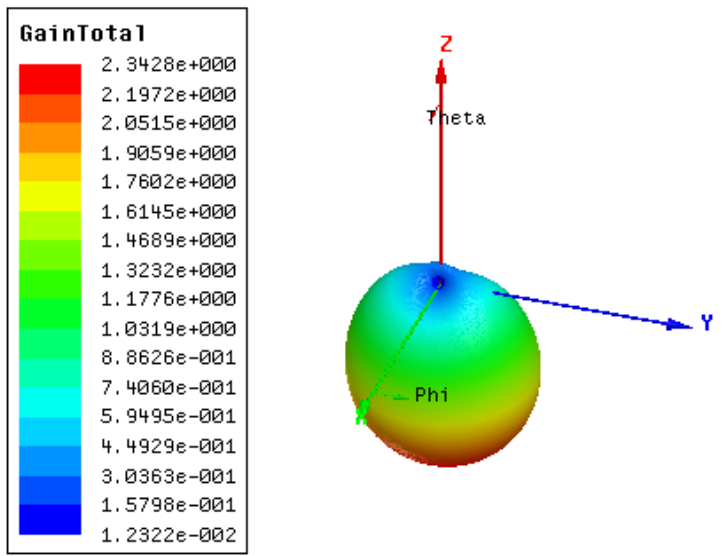

Figure 15. Antenna gain without head. 


\subsubsection{Probe Feed Patch Antenna (Figures 16,17)}
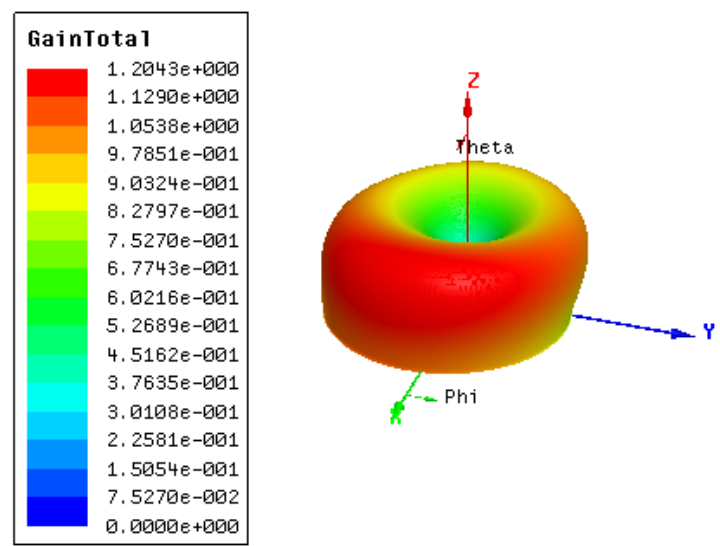

Figure 16. Antenna gain in presence of head.

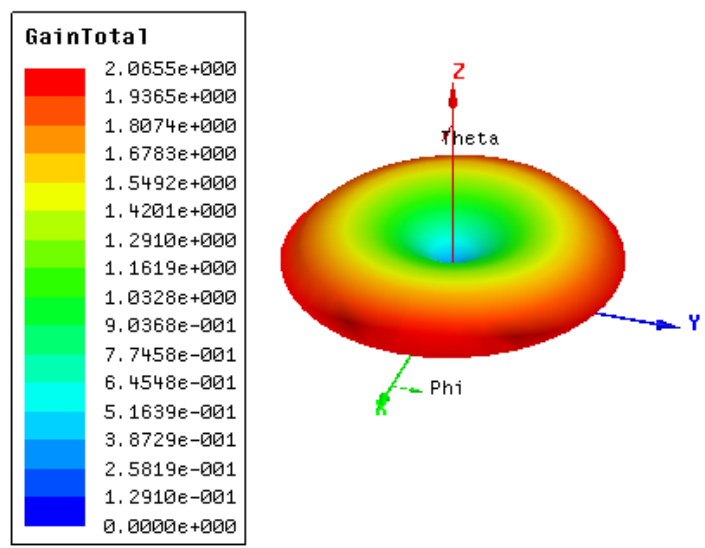

Figure 17. Antenna gain without head.

\section{Conclusion}

In this paper the value of effect of EM has been simulated and described. The paper has been considered that human tissues are interacted with EM and their relationship is in both way, i.e. antenna and human head affects on each other. For example gain of antenna is changing with or without presence of head. The results show that the presence of head reduces the gain of antenna (both antenna).

SAR and E-strength field have been shown in presence of both antennas. The results show that maximum value (red color) for these models happen where the antenna is normal toward to the human model. (In the HFSS software the colors show the amplitude and strength of parameters).

Also there is some problem with these kinds of simulations in real conditions. In real condition the materials of human phantoms are in jell or liquid and it is not possible to simulate three layers easily, so more study have to done.

\section{References}

1. Lak A, Oraizi H. Evaluation of SAR distribution in six-layer human head model. International Journal of Antennas and Propagation;. 2013. p. 8.

2. Kohler S, Ticaud N, Iordache MM, Moisescu MG, Savopol T, Leveque P, Arnaud-Cormos D. Setup for simultaneous microwave heating and real-time spectrofluorometric measurements in biological systems. Progress in Electromagnetics Research. 2014; 145:229-40.

3. Chou CK, Bassen H, Osepchuk J, Balenzo J, Petersen R, Meltz M, Cleveland R, Lin JC. Radiofrequency electromagnetic exposure: tutorial review on experimental dosimetry. Bioelectromagnetics. 1996; 17(3):195-203.

4. Salovarda M, KumarT. Temperature distribution inside GTEM-cell for biomedical experiments. Technicki glasnkik. 2007 Oct; 1(1-2):16-9.

5. Vecchia P. International commission on non-ionizing radiation protection. 2007; 92(6):513.

6. Gabriel C. The dielectric properties of tissues. In: Radiofrequency radiation dosimetry and its relationship to the biological effects of electromagnetic fields. Nato science series. High Technology. 2000; 82:75-84.

7. Mahapatra S, Dey TK. Effects of electromagnetic radiation on bio-objects. 2005. p. 1-4.

8. Royal Society of Canada 1999. A review of the potential health risks of radiofrequency fields from wireless telecommunication devices. An Expert Panel Report prepared at the request of the Royal Society of Canada for Health Canada, Ottawa, Royal Society of Canada. RSC.EPR 99-1.

9. Kues HA, Monahan JC, D’Anna D, McLeod DS, Lutty GA, Loslov S. Increased sensitivity of the non-human primate eye to microwave radiation following opthalmic drug pretreatment. Bioelectromagnetics. 1992; 13(5):379-93.

10. Pattanaik S. Biological effects of RF/ MW radiations on human. Archives of Applied Science Research. 2012; 4(1):381-7.

11. Zamanian A, Hardiman C. Electromagnetic radiation and human health: A review of sources and effects. Fluor Corporation, Industrial and Infrastructure Group. 2005. p. 16-26.

12. Lin JC. The blood-brain barrier, cancer, cell phones and microwave radiation. IEEE Antennas and Propagation Magazine. 2001 Jun; 43(3):141-3.

13. Lin JC. Microwave radiation and leakage of albumin from blood to brain. IEEE Microwave Magazine. 2004 Sep; 5(3):22-8.

14. Sage C. An overview of radiofrequency/microwave radiation studies relevant to wireless communications and data. International Conference on Cell Tower Siting, Salzburg, Austria. Land Salzburg-Landessanitatsdirektion Umweltmedizin, Federal State of Salzburg Public Health 
Department, Environmental Health Unit. 2000 Jun 7-8. p. 90-105

15. Elder JA, Chou CK. Auditory response to pulsed radiofrequency energy. Bioelectromagnetics. 2003; 24(S6):162-73.

16. Habash RWY. Bioeffect and therapeutic applications of electromagnetic energy. USA: Taylor Francies and Group; 2006.

17. Kato M. Electromagnetics in biology. Japan: Springer; 2006.

18. Marianna T, Laputková G, Sabo J, Živčák J. The effect of high-frequency electromagnetic field on molecular melting profile of human breast adenocarcinoma MCF-7 cell DNA. Biomedical Research. 2013; 24(4):469-75.

19. Lak A. Human health effects from radiofrequency and microwave fields. J Basic Appl Sci Res. 2012; 2(9):9446-53.

20. HFSS v10 User Guide;. 2005. Available from: anlage.umd. edu/HFSSv10UserGuide.pdf

21. Garg TK, Gupta SC, Pattnaik SS. Metamaterial loaded frequency tunable electrically small planar patch antenna. 2014 Nov; 7(11):1738-43. 